\title{
Deterministic Polishing of Replicating Grazing-Incidence Mandrels
}

Jacqueline Davis, Patrick Champey, Jeff Kolodziejczak and Charles Griffith

NASA Marshall Space Flight Center, Astrophysics Office, MSFC/ZP12, Huntsville, AL 35812

\begin{abstract}
In an effort to manufacture high-angular-resolution, grazing-incidence, x-ray optics, Marshall Space Flight Center (MSFC) is taking measures to improve its electroformed replicated optics. A key development is the use of computer-numerical control (CNC) polishing to deterministically improve the surface of electroless nickel mandrels used to replicate grazingincidence optics. Metrology, control software and polishing parameters must function together seamlessly to reach the specifications required to replicate sub-arcsecond optics. Each change in polishing parameters effects the wear pattern of the polishing head. Using Richardson-Lucy deconvolution, the controller software fits the wear pattern to metrology data to calculate the changing feedrates across the mandrel. Here we present an overview of our process, and early results showing the effectiveness of deterministic polishing for replicated optics.
\end{abstract}

Keywords: deterministic polishing, grazing-incidence, $x$-ray, optics

\section{BACKGROUND}

From Chandra to ART-XC to IXPE, NASA Marshall Space Flight Center (MSFC) continues to develop new technologies to improve grazing-incidence, $\mathrm{x}$-ray optics. Chandra has very high angular resolution at less than an arcsecond and has produced vast amounts of valuable scientific data for the last 20 years. ART-XC, an instrument aboard Spectrum-Rontgen-Gamma, saw first light in July of this year. Much progress has been made in an effort to increase the collecting area of the x-ray optics by densely-populating the nested shells. Figure 1 shows the Chandra mirror assembly side-by-side with the ART-XC mirror assembly developed at MSFC [1, 2, 3, 4].

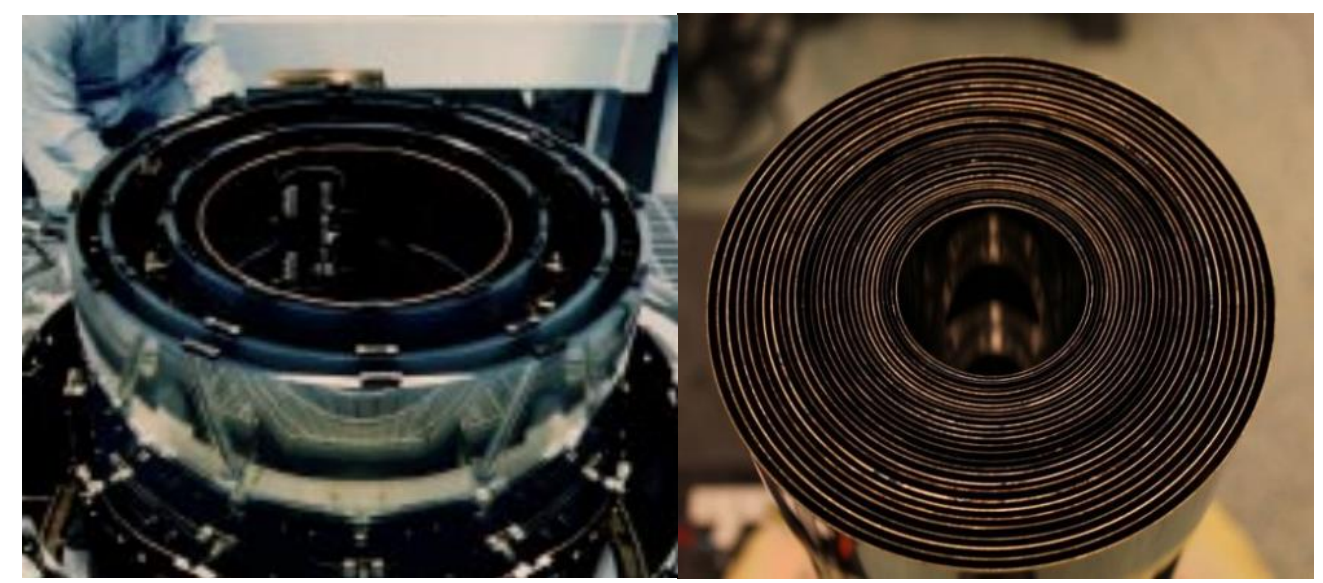

Figure 1: left: Chandra mirror assembly [1]; right: ART-XC mirror assembly.

The technology objective of the x-ray astronomy group at NASA MSFC is to develop new methods of manufacturing Chandra-like optics that maintain its angular resolution and increase the field and the collecting area. The direction of the $\mathrm{x}$-ray group at MSFC is to maintain the full-shell, grazing-incidence optics of Chandra but develop methods to denselypopulate the nested shells in order to increase the collecting area within the same general volume. $[1,2,4,5]$. 
A comparison of the specifications and general size of Chandra and ART-XC is shown below. The collecting area per volume of ART-XC is approximately 10 times that of Chandra.

- Chandra optics

- Zerodur

- 0.5 " HPD

- 4 p-h shell pairs

- outer radius $=0.60 \mathrm{~m}$

- length $=1.66 \mathrm{~m}$

- collecting area $\sim 15 \mathrm{~m}^{2}$

- $\quad$ ART-XC optics

- nickel cobalt

- 25" HPD

- 28 p-h shells

- outer radius $=0.065 \mathrm{~m}$

- length $=0.58 \mathrm{~m}$

- collecting area $\sim 1 \mathrm{~m}^{2}$
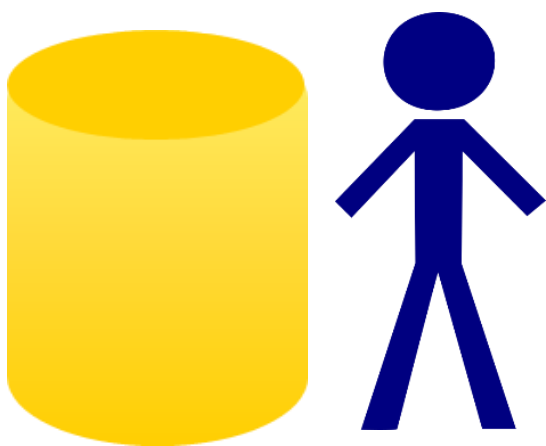

Figure 2: Left: outer Chandra shell; Center: 5'6" stick figure; Right: outer ART-XC shell.

The manufacturing process used at MSFC to manufacture the ART-XC shells to electroform nickel optics has been used at MSFC for a variety of applications for almost 25 years (Figure 3). The shells are electrodeposited onto a figured mandrel and released by cooling. In order to densely populate the shells, the electroformed nickel shells are much thinner than the Chandra shells, which also makes them more susceptible to figure errors. The angular resolution for the ART-XC shells is 25" HPD, however, the desired angular resolution is that of the Chandra optics, 0.5" HPD [6].

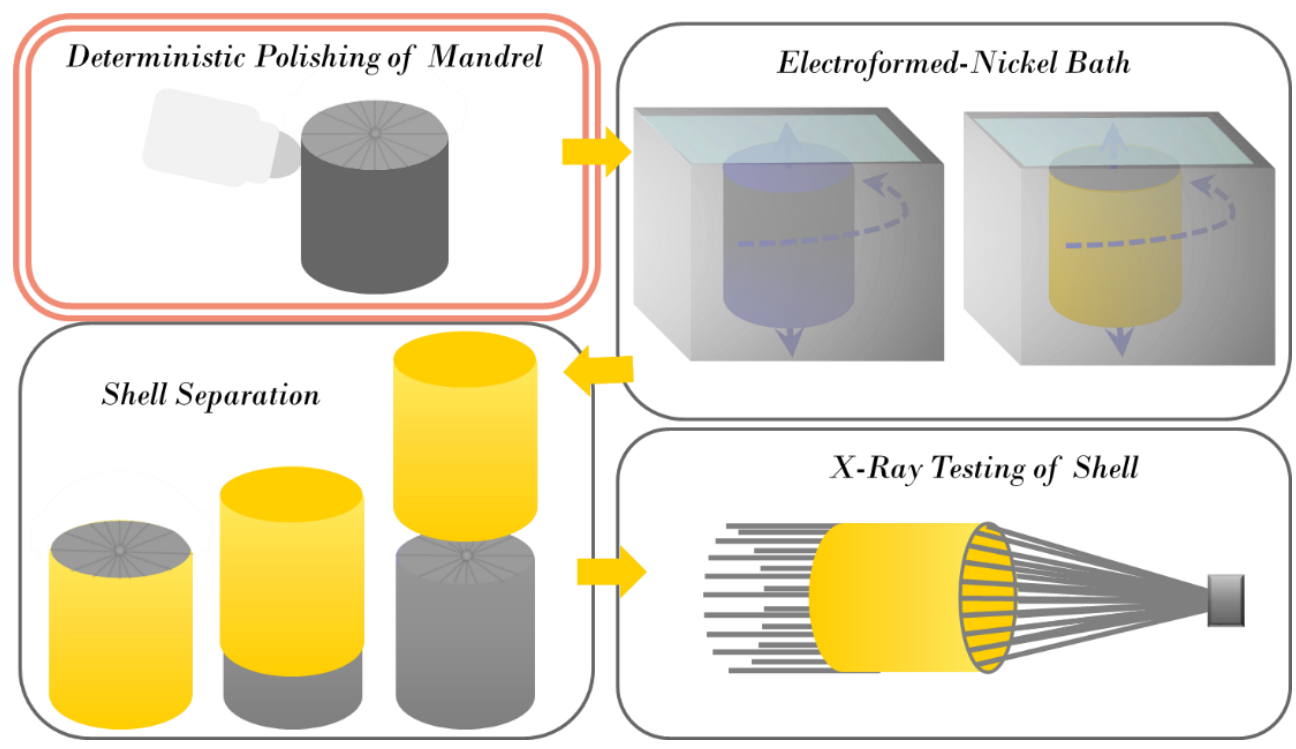

Figure 3: MSFC electroformed nickel optics manufacturing process, including deterministic polishing of mandrel

As discussed in this paper, deterministic polishing of the mandrel is a viable method to reduce error in the electroformed nickel shells. Investigating improvements to each step of the electrodeposition process, along with other methods of manufacturing, MSFC is moving toward high-resolution, high-collecting area, full-shell, grazing-incidence optics [4, 5, 7]. 


\section{PREMISE OF LATERAL DETERMINISTIC POLISHING}

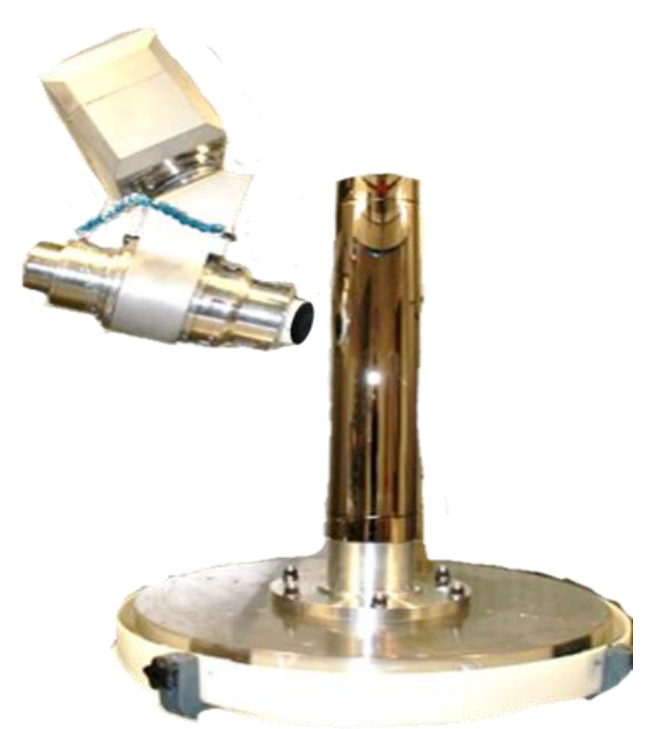

Figure 4: MSFC deterministic polishing of mandrel.
Traditionally, lap polishing has been used to shape and refine astronomical optics. This method of craft polishing is not deterministic, and requires many iterations of measurements and polishing.

Wikipedia defines a deterministic system as a system whose initial state is known exactly and whose future state system can be theoretically be predicted [8]. In order to create a deterministic polishing system, the precision in the following areas are necessary:

- $\quad$ initial state

- $\quad$ prediction characteristics

- fitting algorithm

The concept of computer-controlled polishing began at the Optical Science Laboratory (OSL) at University College London to polish large optics for astronomy, and the subsequent spin-off company, Zeeko, now has 7-axis polishing machines available for purchase. The machine enables a control system of polishing, however, the initial state, prediction characteristics and the fitting algorithm define whether or not the polishing system is deterministic.

Polishing using the Zeeko polisher has proven very effective on flat optics, and the ability to perform freeform surface machining can provide prescription accuracy $[7,9,10]$. However, much development is necessary to perform is comparable precision on cylindrically-shaped optics.

\subsection{Initial State: Metrology}

In order to attain a precise initial state of the deterministic polishing system, high resolution metrology data must be collected. Current development is underway at MSFC to increase the resolution of metrology of mandrels and shells. The method shown in Figure 5 uses a Computer-Generated Hologram (CGH) in line with an interferometer to collect data across a sector of the mandrel. To date, the methods for measuring mandrels is stitching data of meridians spaced around $15^{\circ}$ apart. Increasing the amount of data collected circumferentially around the mandrel increases the data resolution over the entire surface.

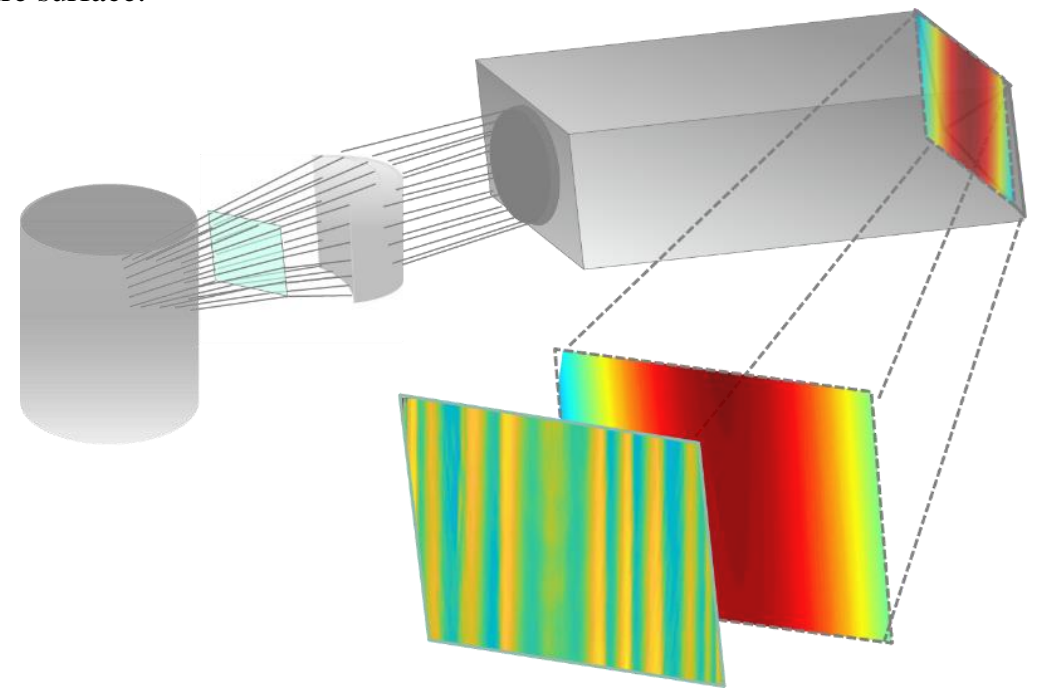

Figure 5: mandrel metrology layout 
The beam from the interferometer passes through the CGG, hits the mandrels, then passes back through the CGH creating a shift between the "ideal" surface and the "actual" surface. The idea is to "flatten" out the data with the CGH, creating an interferogram of the deviation of the mandrel from its prescription.

Most of the alignment error will be reduced when aligning the system, however, the remaining error must be subtracted from the data. The surface distortion must be calculated using the data collected by the interferometer, which is actually the alignment deviation at the plane of the $\mathrm{CGH}$. Therefore, any mis-alignments of the mandrel (x-direction, $\mathrm{y}$-direction, $\mathrm{z}$ direction, $\mathrm{xy}$-direction, $\mathrm{xz}$-direction, $\mathrm{yz}$-direction) are transformed to a radial deformation, $\delta \mathbf{r}$. The equation below is the radial deformation at the plane of the CGH in terms of mis-alignment of each point $\left(\mathbf{x}_{\mathbf{0}}, \mathbf{y}_{\mathbf{0}}, \mathbf{z}_{\mathbf{0}}\right)$ on the mandrel. The equation was expanded using the Taylor series, creating an ortho-normal polynomial set to fit and subtract alignment error from each data set.

$$
\delta \mathbf{r}=\left[\left(\mathbf{x}_{\mathbf{o}}+\mathrm{a} \mathbf{y}_{\mathbf{o}}+\mathrm{b} \mathbf{z}_{\mathbf{o}}+\mathrm{d}\right)^{2}+\left(\mathbf{y}_{\mathbf{o}}+\mathrm{f} \mathbf{x}_{\mathbf{o}}+\mathrm{g} \mathbf{z}_{\mathbf{o}}+\mathrm{h}\right)^{2}+\left(\mathbf{z}_{\mathbf{o}}+\mathrm{k} \mathbf{x}_{\mathbf{0}}+\mathrm{m} \mathbf{y}_{\mathbf{o}}+\mathrm{n}\right)^{2}\right]^{1 / 2}-\left(\mathbf{x}_{\mathbf{0}}{ }^{2}+\mathbf{y}_{\mathbf{o}}{ }^{2}+\mathbf{z}_{\mathbf{o}}{ }^{2}\right)^{1 / 2}
$$

\section{$1^{\text {st }}$ order Taylor Series expansion:}

x-direction: $\mathrm{c}_{1} * \mathrm{x}_{0} /\left(\mathrm{x}_{\mathrm{o}}{ }^{2}+\mathrm{y}_{\mathrm{o}}{ }^{2}+\mathrm{z}_{\mathrm{o}}{ }^{2}\right)^{1 / 2}$

y-direction: $\mathrm{c}_{2} * \mathrm{y}_{\mathrm{o}} /\left(\mathrm{x}_{\mathrm{o}}^{2}+\mathrm{y}_{\mathrm{o}}^{2}+\mathrm{z}_{\mathrm{o}}^{2}\right)^{1 / 2}$

z-direction: $\mathrm{c}_{3} * \mathrm{z}_{\mathrm{o}} /\left(\mathrm{x}_{\mathrm{o}}{ }^{2}+\mathrm{y}_{\mathrm{o}}{ }^{2}+\mathrm{z}_{\mathrm{o}}{ }^{2}\right)^{1 / 2}$

xy-direction: $\mathrm{c}_{4} *\left(\mathrm{x}_{\mathrm{o}} * \mathrm{y}_{\mathrm{o}}\right) /\left(\mathrm{x}_{\mathrm{o}}{ }^{2}+\mathrm{y}_{\mathrm{o}}{ }^{2}+\mathrm{z}_{\mathrm{o}}{ }^{2}\right)^{1 / 2}$

xz-direction: $\mathrm{c}_{5} *\left(\mathrm{x}_{\mathrm{o}} * \mathrm{z}_{\mathrm{o}}\right) /\left(\mathrm{x}_{\mathrm{o}}^{2}+\mathrm{y}_{\mathrm{o}}^{2}+\mathrm{z}_{\mathrm{o}}^{2}\right)^{1 / 2}$

yz-direction: $\mathrm{c}_{6} *\left(\mathrm{y}_{\mathrm{o}} * \mathrm{z}_{\mathrm{o}}\right) /\left(\mathrm{x}_{\mathrm{o}}^{2}+\mathrm{y}_{\mathrm{o}}^{2}+\mathrm{z}_{\mathrm{o}}^{2}\right)^{1 / 2}$

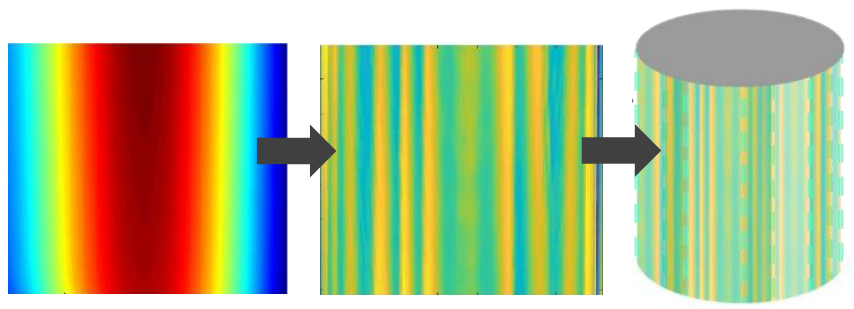

Figure 6: alignment subtraction and stitching of metrology

Once the data is collected and alignment errors subtracted, the mandrel will be rotated. Each sector of the mandrel will be measured and stitched for the entire surface of the mandrel.

\subsection{Prediction Characteristics: Polishing Parameters}

In order to predict the effect of polishing on the polishing surface, the effect of polishing parameters must be thoroughly characterized. Using the Zeeko polishing machine, its input parameters are defined as follows [10]:

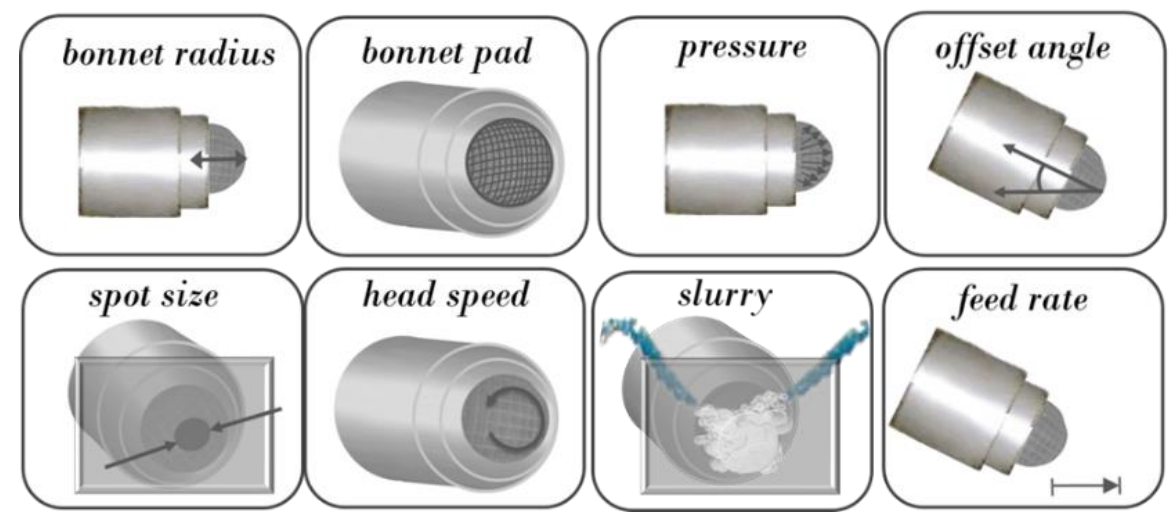

Figure 7: deterministic polishing parameters

- bonnet radius: radius of curvature of inflated polishing tool

- $\quad$ spot size: tool contact area when pressed against the work-piece

- bonnet pad: type and contoured shape of pad adhered to polishing tool

- headspeed: speed of rotation of the polishing head

- bonnet pressure: internal pressure of the tool 
- slurry: type and size of abrasive applied between polishing tool and optical surface

- offset angle: tool angle of attack

- feedrate: speed at which the polishing head moves across the part

Craft polishing has taught us about the effect of the polishing parameters in a general sense. However, in a deterministic polishing routine, the exact effect of polishing parameters on the shape of the wear pattern is paramount. A single measurement of the wear pattern with a specific set of parameters can be used to create a deterministic fit, but a more thorough knowledge of various combinations of polishing parameters will create an ability to remove more error [7, 9, $10]$.

\subsection{Fitting Algorithm: Richardson-Lucy Deconvolution}

The fitting algorithm is paramount to creating a deterministic system, applying the effect of the polishing to the initial surface error. The alignment between all of the moving parts is vital. The wear pattern and the initial error must first be put on the same grid, which is created using the point spacing and the track spacing, two of the polishing parameters.
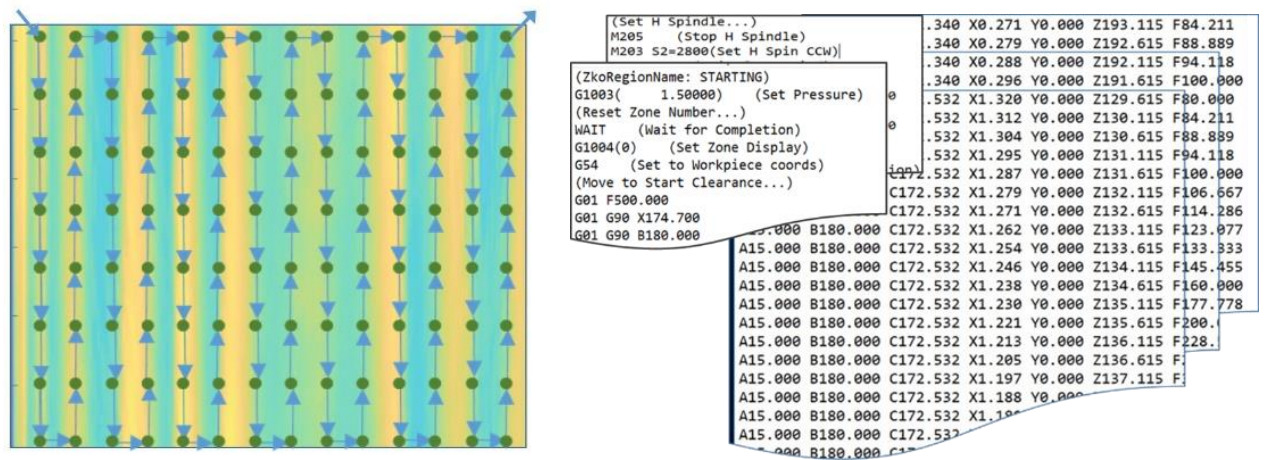

Figure 8: polishing grid on optical surface, resulting custom g-code for polishing run

Because the wear pattern is at times non-linear in its fit to the error, an iterative approach was chosen. The RichardsonLucy Deconvolution is a method used to "de-blur" an underlying image by using a known point source. In this case, the point source is the wear pattern, and the image is the metrology error.

$$
\begin{gathered}
\hat{u}^{(t+1)}=\hat{u}^{(t)} \cdot\left(\frac{d}{\hat{u}^{(t)} \otimes P} \otimes P^{*}\right) \\
\mathrm{P}=\text { wear function } \\
\mathrm{P}^{*}=\text { wear function transpose } \\
\mathrm{d}=\text { metrology error }
\end{gathered}
$$

The resulting information from the fit is a scalar coefficient that determines the feedrate between gridded polishing points.

\section{IMPLEMENTATION OF DETERMINISTIC POLISHING OF MANDRELS}

The deterministic polishing discussed in Section 2 was implemented using a mandrel developed for the MaGIXS sounding rocket mission [34]. 1-dimensional deterministic polishing corrected the mandrel from 16" HPD (after lap polishing) to 6" HPD. Next, one iteration of 2-dimensional deterministic polishing was done. Custom software and polishing processes were developed, including custom g-code files, alignment and registration improvements, and polishing parameter influence on wear patterns. 


\subsection{Metrology}

The metrology system described in Section 2.1 was set up using a CGH matching the MaGIXS prescription. An interferogram was taken of the mandrel using the $\mathrm{CGH}$, and the alignment errors were subtracted. Figure 9 shows the resulting error between $33-57^{\circ}$.
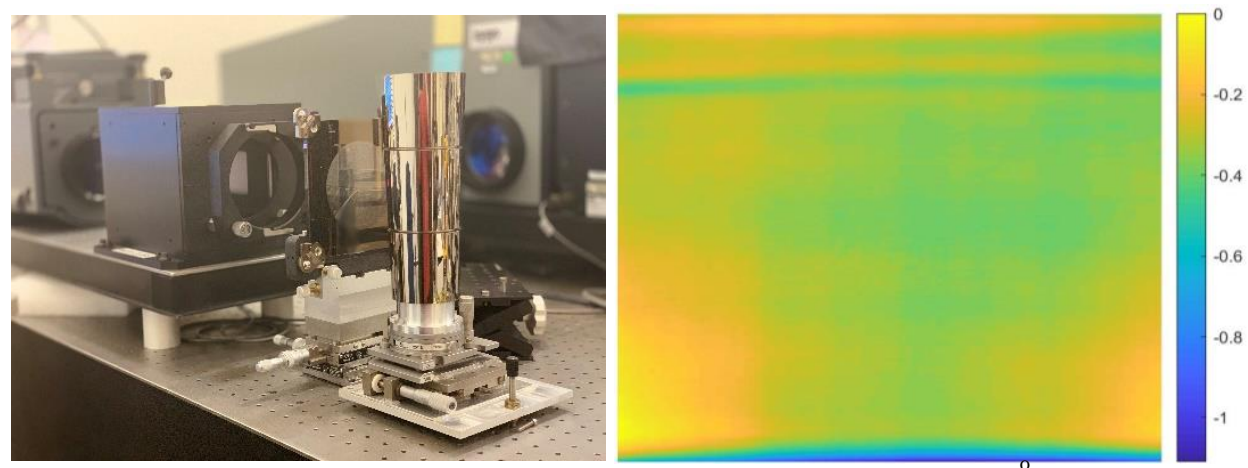

Figure 9: surface error $(\mu \mathrm{m})$, center section, 33-57 .

Currently, this is the only section measured and polished with the 2-dimensional deterministic polishing routine.

\subsection{Parameter Characterization}

The polishing parameters shown in Figure 10 were chosen for the implementation of the 2-dimensional polishing run, and the resulting wear pattern measured.

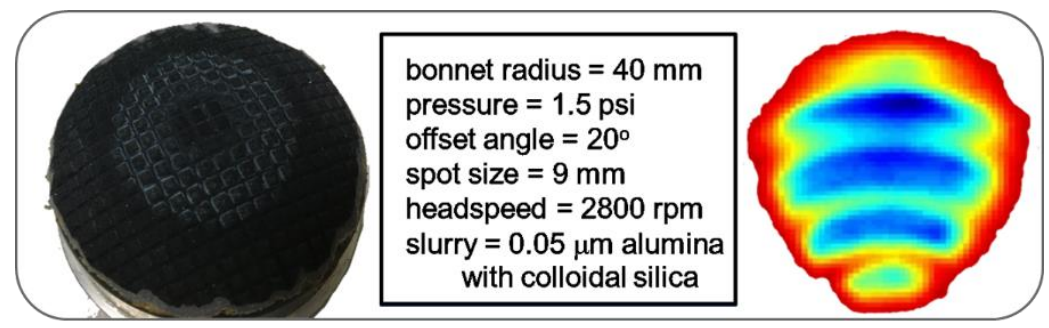

Figure 10: polishing parameters and resulting wear pattern

Because the polishing feed must be applied in a grid-like format, as shown below in Figure 11, the wear rate is calculated for a given spacing.
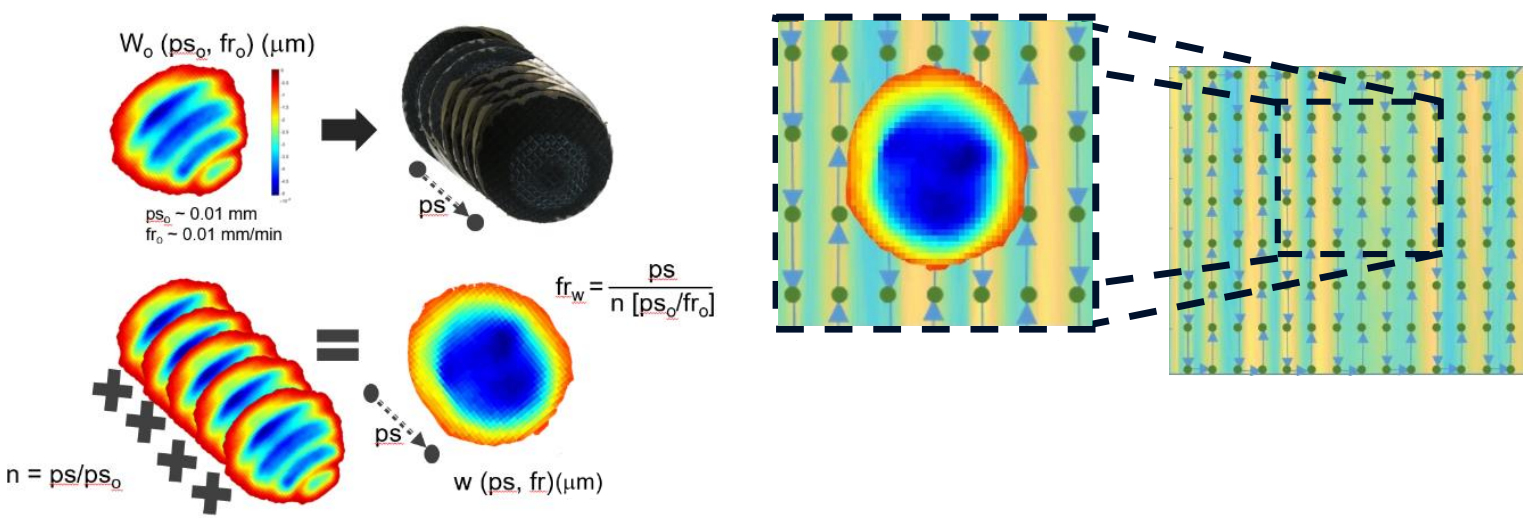

Figure 11: left: wear function calculation; right: wear function on polishing grid.

The "instantaneous" wear pattern with the desired polishing parameters, $\mathrm{w}_{\mathrm{o}}$, is measured at a short point spacing, $\mathrm{ps}_{\mathrm{o}}$, and a slow feedrate, $\mathrm{fr}_{\mathrm{o}}$. The short point spacing and slow feedrate make the signal to noise ratio high in order to get an 
accurate measurement of the wear pattern. The point spacing is also very small relative to the point spacings used in polishing runs, hence the name "instantaneous" wear pattern.

To calculate the wear function, the measured wear pattern is added $\mathrm{n}$ times across the distance of one point spacing $\left(\mathrm{ps} / \mathrm{ps}_{\mathrm{o}}\right)$. The resulting wear function, $\mathrm{fr}_{\mathrm{w}}$, is a map of the depth removed per second over a given point spacing.

\subsection{Richardson-Lucy Deconvolution}

Using Richardson-Lucy Deconvolution, the wear function described in Section 3.2 was applied to the initial surface error described in Section 3.1. The wear function and the surface error are interpolated to the polishing grid points on the optical surface described in Section 2.3. Great care is taken in this step to insure proper registration and alignment.

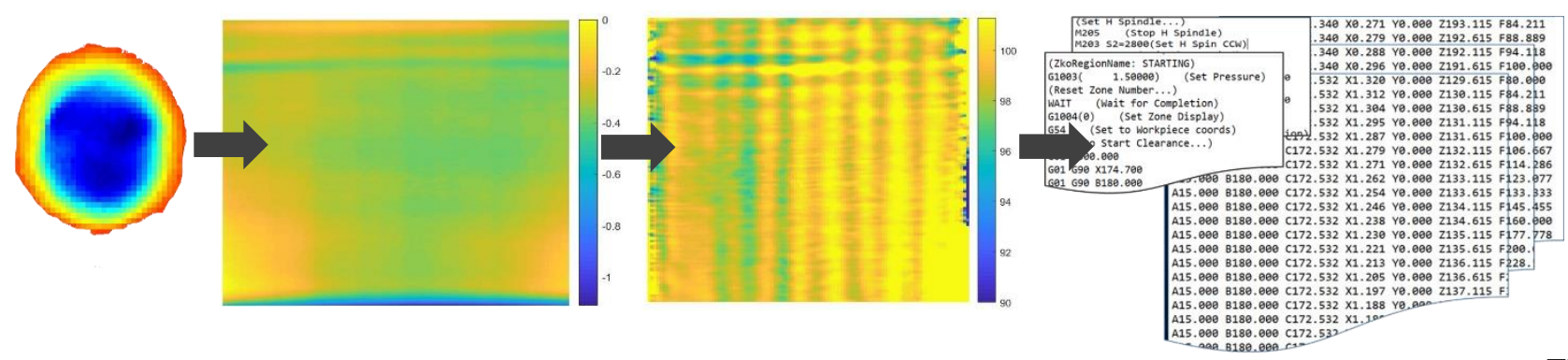

Figure 12: Richardson-Lucy fit of wear function to MaGIXS mandrel error $(\mu \mathrm{m})$, resulting in feedrate $(\mathrm{mm} / \mathrm{min})$ and The exported to a custom g-code to input into polishing machine.

output of the fit is a scalar of the wear function, $\mathrm{fr}_{\mathrm{w}}$, described in Section 2.3. The feedrate between each grid point was computed and exported to a custom g-code.

\section{2-DIMENSIONAL DETERMINISTIC POLISHING RESULTS}

\subsection{Registration and Alignment of Mandrel}

The alignment custom g-code was run to measure the tilt and decenter of the mandrel, and the registration custom g-code was run in order to define a global coordinate system. The tilt, decenter and global coordinate system is used in conjunction with the track spacing, point spacing and prescription of the mandrel to create a polishing grid on the surface of the mandrel. The custom registration routine uses a probing function in the $\mathrm{z}$-direction in order to set the global $\mathrm{z}_{\mathrm{o}}$ at the lower groove in the mandrel. Another custom $\mathrm{g}$-code is run to probe in the $\mathrm{x}$-direction and set $\mathrm{x}_{\mathrm{o}}$ at the surface of the mandrel; this will function as the $r_{o}$ for subsequent g-codes. Figure 13 shows the information exported from the alignment and registration runs. The decenter and tilt are calculated from the data. The alignment and registration is used to create the polishing grid, which, along with the feedrates from Section 3.3, are used to create the custom polishing g-code.

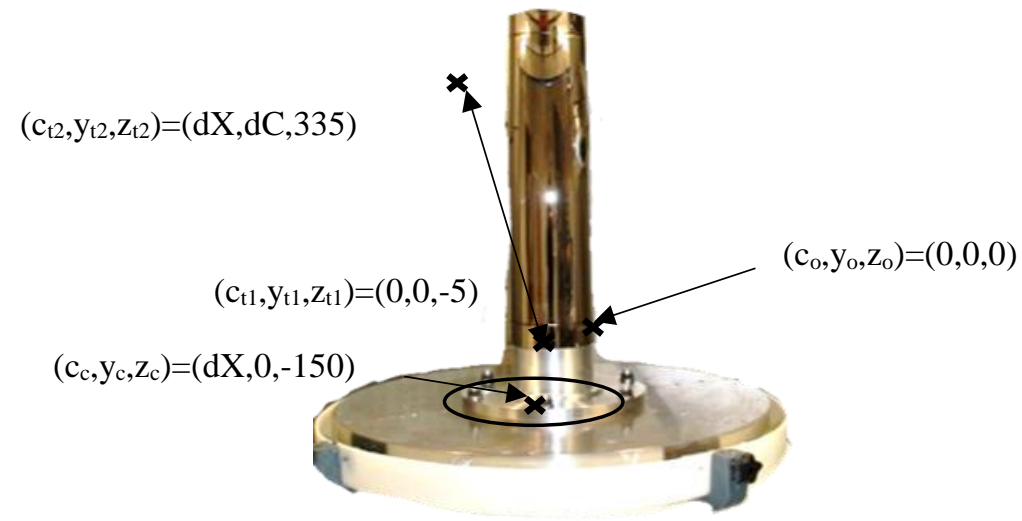

Figure 13: alignment and registration of mandrel 


\subsection{2-Dimensional Deterministic Polishing Results of Mandrel}

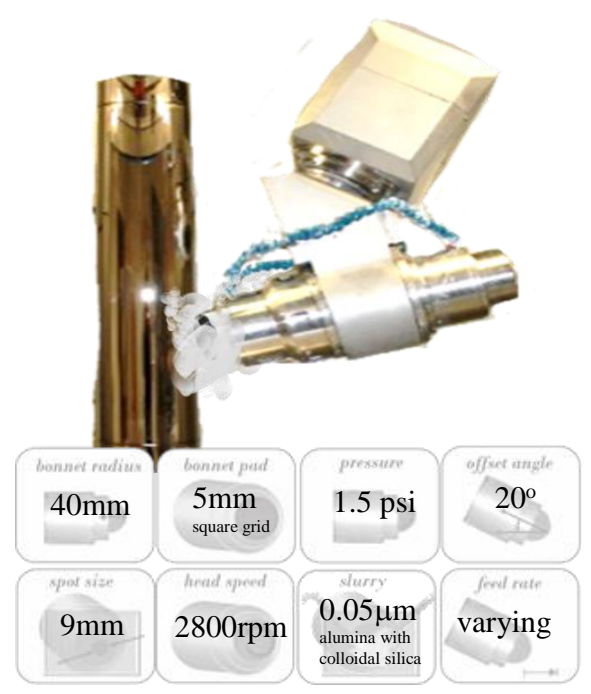

Figure 14: top: slurry applied to mandrel during polishing; bottom: polishing parameters applied during polishing.

Combining the polishing parameters used for the wear function (Figure 14), the registered and aligned polishing grid discussed in Section 4.1, and the feedrates determined in Section 3.3, the custom polishing g-code was generated.

As shown in Figure 14, the slurry is composed of $0.05 \mu \mathrm{m}$ alumina silica and is applied to the surface of the mandrel, creating an abrasive between the pressurized bonnet and the mandrel. The bonnet was pressurized at $1.5 \mathrm{psi}$ with a $5 \mathrm{~mm}$ square grid pad adhered to it. The $40 \mathrm{~mm}$ bonnet, rotating at 2800rpm, was pushed into the mandrel surface at $20^{\circ}$, and created a $9 \mathrm{~mm}$ spot size on the surface.

After the polishing run, the mandrel was put in line with the CGH in front of the interferometer, shown in Figure 15. The interferogram was taken, and the alignment errors were removed.

The slope error between $45^{\circ}$ and $60^{\circ}$ improved from 12" HPD to 4" HPD. Figure 16 shows the resulting measurement in comparison with the initial error. The surface root-mean-square (RMS) was initially $0.1 \mu \mathrm{m}$ and after the polishing run was $0.05 \mu \mathrm{m}$ RMS.

The entire polished surface, between $33^{\circ}$ and $60^{\circ}$, improved from $12^{\prime}$ HPD to $6^{\prime \prime ~ H P D, ~ h o w e v e r, ~ a ~ g l o b a l ~ t i l t ~ w a s ~ i n d u c e d ~}$ into the mandrel. The section of the mandrel between $45^{\circ}$ and $60^{\circ}$ showed significantly more improvement than between $33^{\circ}$ and $45^{\circ}$. We will investigate the cause of the discrepancy, but we believe the error is due to an alignment issue.

We consider these results very promising, especially given it was the first run of a 2-dimensional deterministic polishing run. The path forward is to investigate the cause of the global induced error. The initial studies will be testing the alignment and registration at each step of the process. We plan to use highly calibrated fiducials on the mandrel, and we are investigating ways to validate the metrology. Collecting more polishing data and analyzing the wear pattern reproducibility will also provide more information about errors in the system.

Next, we will collect data around the entire circumference of the mandrel, stitch the data, and polish the entire surface. We have already replicated shells from the mandrels that were deterministically polished using 1-dimensional metrology data, and we will do the same for the mandrels polished with 2 dimensional data. Then, the process can be validated using $\mathrm{x}$-ray testing.

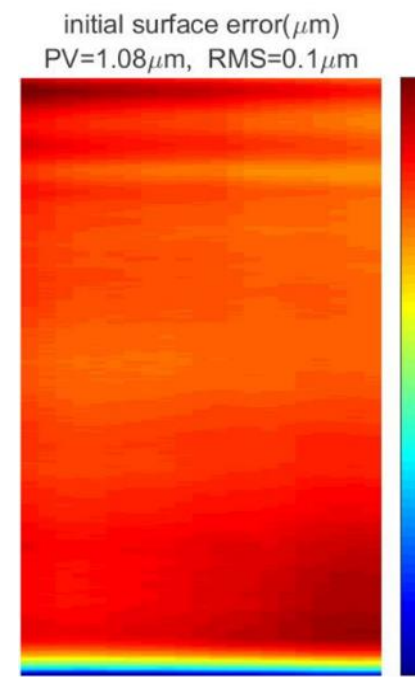

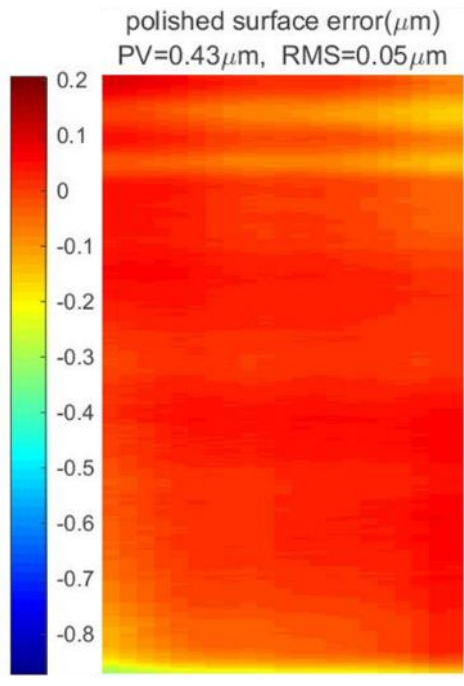

Figure 16: left: initial surface error, 12" HPD; right: polished surface error, 4" HPD. 


\section{SUMMARY}

In moving toward the goal of sub-arcsecond HPD full-shell, grazing-incidence optics, MSFC has inserted deterministic polishing into its existing replicated optics process. By improving the surface figure of the replicating mandrel, the resulting shells inherently improve in angular resolution. Because the Zeeko polishing machine was not conducive to lateral polishing for cylindrical parts, custom software and polishing processes were developed, including custom g-code files, alignment and registration improvements, polishing parameter influence on wear patterns.

The process was implemented on the MaGIXS sounding rocket mandrel, improving its surface slope error from 16" HPD with lap polishing to 6" HPD using 1-dimensional deterministic polishing. To improve the process even further towards the goal of 1" HPD, initial stages of 2-dimensional deterministic polishing were done and reported.

A single 2-dimensional polishing run was implemented on the MaGIXS mandrel. Because azimuthal metrology was averaged around the circumference for the 1-dimensional deterministic polishing, high resolution metrology data was necessary for the 2-dimensional run. Therefore, a new method was developed. A CGH was put in front of the interferometer in order to gain more information across the circumference of the mandrel. A method of subtracting alignment error was also necessary. Therefore, ortho-normal functions were calculated and subtracted from the metrology data. Initial findings show that the 2-dimensional polishing shows is a viable method for correcting surface error.

Next, we will create fiducials in order to make alignment and registration more precise. Then, a method to stitch the sectors around the circumference of the mandrel will be developed in order to polish the full mandrels. Also, further investigations into the impact of the polishing parameters on the wear functions will be done in order to reduce surface error even further.

\section{REFERENCES}

[1] Weisskopf, M.C., Aldcroft, T.L., Bautz, M., Cameron, R.A., Dewey, D., Drake, J.J, Grant, C.E., Marshall, H.L., and Murray, S.S, “An Overview of the Performance of the Chandra X-ray Observatory," Exp. Astron. 16, 1-68 (2003).

[2] Weisskopf, M.C., Brinkman, B., Canizares, C., Garmire, G., Murray, S., and Van Speybroeck, L.P., "An Overview of the Performance and Scientific Results from the Chandra X-Ray Observatory," Pub. Astron. Soc. Pac. 114, 1-24 (2002).

[3] Weisskopf, M.C., Tananbaum, H.D., Van Speybroeck, L.P., and O'Dell, S.L., "Chandra X-ray Observatory (CXO): overview," Proc. SPIE 4012, 2-16 (2000).

[4] M. Gubarev, B. Ramsey, J. J. Kolodziejczak, S. L. O'Dell, R. Elsner, V. Zavlin, D. Swartz, M. Pavlinsky, A. Tkachenko, I. Lapshov, "The calibration of flight mirror modules for the ART-XC instrument on board the SRG mission," Proc. SPIE 9144, Space Telescopes and Instrumentation 2014: Ultraviolet to Gamma Ray, 91444U (July 28, 2014).

[5] Roche, J. M., Kolodziejczak, J. L., O’Dell, S. L., Elsner, R. F., Weisskopf, M. C., Ramsey, B. D., and Gubarev, M. V., "Opto-mechanical analyses for performance optimization of lightweight grazing-incidence mirrors," Proc. SPIE 8861, 48 (2013).

[6] Ramsey, B.D., Atkins, C., Gubarev, M.V., Kilaru, K., O’Dell, S.L., "Optics requirements for x-ray astronomy and developments at the Marshall Space Flight Center," Nuclear Instruments and Methods in Physics Research A 616, 110 (2012).

[7] Abigail R. Hooper, Nathan N. Hoffmann, Harry W. Sarkas, John Escolas, Zachary Hobbs, "Deterministic polishing from theory to practice," Proc. SPIE 9633, Optifab 2015, 96330C (11 October 2015).

[8] Wikipedia contributors. "Deterministic system." Wikipedia, The Free Encyclopedia. Wikipedia, The Free Encyclopedia, 1 Apr. 2019. Web. 5 Sep. 2019.

[9] Liang, F., Zhao, J., Ji, S., \& Lu, L. (2018). Deterministic polishing of freeform optical surface by iterative intersection tool path in an off-axial three-mirror anastigmat imaging system. Proceedings of the Institution of Mechanical Engineers, Part B: Journal of Engineering Manufacture, 232(5), 816-826.

[10] Richard G. Bingham, David D. Walker, Do-Hyung Kim, David Brooks, Richard Freeman, Darren Riley, "Novel automated process for aspheric surfaces," Proc. SPIE 4093, Current Developments in Lens Design and Optical Systems Engineering, (24 October 2000). 
[11] Ken Kobayashi, Jonathan Cirtain, Leon Golub, Amy Winebarger, Edward Hertz, Peter Cheimets, David Caldwell, Kelly Korreck, Brian Robinson, Patrick Reardon, Thomas Kester, Charles Griffith, Mark Young, "The Marshall Grazing Incidence X-ray Spectrograph (MaGIXS)," Proc. SPIE 8147, Optics for EUV, X-Ray, and GammaRay Astronomy V, 81471M (6 October 2011). 